\section{Plasma cystatin C: a better marker of GFR than serum creatinine?}

Serum creatinine level is generally used as a surrogate marker for glomerular filtration rate (GFR), but is affected by patient-specific factors including diet, sex, ethnicity and age. Complex prediction equations are necessary to correct for these factors. A Swedish group has found that GFR can be estimated solely from the concentration of cystatin $\mathrm{C}$ in plasma.

Data were analyzed from 536 consecutive patients (age 0.3-93 years; 274 males), for whom GFR had already been measured accurately using an invasive technique. Prediction equations for GFR were constructed on the basis of plasma cystatin $C$ levels, and results were compared with those from the simplified 4-parameter Modification of Diet in Renal Disease equation (for adults) and the Schwartz and Counahan-Barratt equations (for children). The cystatin-C-based prediction equation developed using data from the adult cohort estimated GFR in adults at least as accurately as with current prediction equations, and performed equally well at predicting GFR in children aged $>14$ years. In children aged $<14$ years, the equation required a consistent adjustment factor.

Prediction equations are highly populationdependent, and the fact that this single-center study population included healthy individuals and did not include any African Americans or children aged $<0.3$ years might have affected the results; however, the study provides good support for the use of plasma cystatin $\mathrm{C}$ as a marker for GFR.

Caroline Barranco

Original article Grubb A et al. (2005) Simple cystatin-Cbased prediction equations for glomerular filtration rate compared with the modification of diet in renal disease prediction equation for adults and the Schwartz and the Counahan-Barratt prediction equations for children. Clin Chem 51: 1420-1431

\section{Impact of HAART on the ESRD epidemic is confounded by HIV infection rates}

The epidemic of HIV-associated end-stage renal disease (HIV + ESRD) is projected to worsen over the next 15 years despite the use of highly active antiretroviral therapy (HAART), according to a retrospective analysis of epidemiologic data from the US. Previous claims that the problem is under control might be dangerously misleading.

As most sufferers of HIV-associated nephropathy are black, a predictive model was developed using data from black AIDS and ESRD populations. Inclusion of data from 4 years before to 7 years after the introduction of HAART, together with infection rate, permitted assessment of the effect of HAART on the prevalence of HIV + ESRD to date. The validated model was then used to estimate prevalence through to 2020.

HAART was seen to have had a positive impact on AIDS growth rate since its introduction in 1995. It has also slowed progression to ESRD by at least $38 \%$ and reduced ESRDassociated mortality by $67 \%$. Unfortunately, the model predicts that current linear rates of HIV infection will negate these positive effects, causing the HIV + ESRD population to grow steadily over the next 15 years. This increase will manifest even if the efficacy of HAART in blocking progression to ESRD reaches $95 \%$ or ESRD-associated mortality decreases to $10 \%$.

The authors conclude that the HIV+ESRD epidemic is far from reaching a plateau. They recommend more widespread implementation of HAART and an emphasis on preventing new infections.

\section{Rachael Williams}

Original article Schwartz EJ et al. (2005) Highly active antiretroviral therapy and the epidemic of HIV+ end-stage renal disease. J Am Soc Nephrol 16: 2412-2420

\section{Suboptimal physician awareness is hampering early detection of chronic kidney disease}

Cross-sectional analysis of data from a large regional laboratory in the US has revealed worryingly low rates of serum creatinine testing. Combined with the insensitivity of diagnostic codes for chronic kidney disease (CKD) that also came to light, this study highlights an urgent need for improved physician awareness.

The laboratory in Ohio, which serves 35 million people, processed over 4 million tests for 277,111 adults aged 40 years. The serum creatinine level of a mere $19 \%$ was assessed during the 1-year study period. Of those tested, $30 \%$ had CKD as defined by estimated glomerular filtration rate $\left(\right.$ GFR) $<60 \mathrm{ml} / \mathrm{min} / 1.73 \mathrm{~m}^{2}$. Rates of serum creatinine testing were low $(53 \%)$ 